\title{
Singularity formation for complex solutions of the 3D incompressible Euler equations
}

\author{
Russel E. Caflisch ${ }^{1}$ \\ Department of Mathematics, UCLA, Los Angeles, CA 90024, USA
}

Received 30 July 1992

Revised manuscript received 2 December 1992

Accepted 5 December 1992

Communicated by $\mathrm{H}$. Flaschka

\begin{abstract}
Moore's approximation method, first formulated for vortex sheets, is generalized and applied to axi-symmetric flow with swirl and with smooth initial data. The approximation preserves the forward cascade of energy but neglects any backflow of energy. It splits the Euler equations into two sets of equations: one for $u_{+}=u_{+}(r, z, t)$ containing all non-negative wavenumbers (in $z$ ) and the second for $u_{-}=\bar{u}_{+}$. The equations for $u_{+}$are exactly the Euler equations but with complex initial data. Traveling waves solutions $u_{+}=u_{+}(r, z-\mathrm{i} \sigma t)$ with imaginary wave speed are found numerically for this problem. The asymptotic properties of the resulting Fourier coefficients show a singularity forming in finite time at which the velocity blows up.
\end{abstract}

\section{Introduction}

In three-dimensional inviscid, incompressible flow, the vorticity can grow through vortex stretching, and it is possible that singularities (infinite vorticity) may develop in finite time from smooth initial data. The interest in these possible singularities is mathematical, numerical and physical: Singularities in any solutions of the three dimensional Euler equations would prevent establishment of global existence theorems. Special numerical methods would be required for singularities or nearly singular behavior in numerical solutions. Finally, singularity formation may be a primary mechanism for transfer of energy from large to small wavelengths and thus an essential ingredient in the onset of turbulence. Moreover singularities, which can be de-

\footnotetext{
${ }^{1}$ Research supported in part by grant \#DAAL03-91-G0162 from the Army Research Office and grant \#AFOSR 89-0003 from the Air Force Office of Scientific Research.
}

scribed through a small number of parameters, may provide the simplest possible description of a complex flow.

The main analytic result on singularity formation in 3D is that of Beale, Kato and Majda [7], who showed that

$$
\|u(\cdot, t)\|_{s} \leq \Phi\left(\|u(\cdot, 0)\|_{s}, \int_{0}^{t} \sup _{x}\left|\omega\left(x, t^{\prime}\right)\right| \mathrm{d} t^{\prime}\right)
$$

for some smooth function $\Phi$ and for any Sobolev norm $\|\cdot\|_{s}$ with $s \geq 3$. This establishes that if any smoothness is lost at time $t_{*}$, then in fact $\int_{0}^{t} \max _{x}$ $|\omega| \mathrm{d} t^{\prime}=\infty$. A similar result, that loss of analytically implies blow-up of vorticity, was proved by Bardos and Benachour [6]. For inviscid, incompressible flow in two dimensions, an initially smooth velocity field $\boldsymbol{u}(\boldsymbol{x}, 0)$ will stay smooth for all time due to the conservation of vorticity.

The numerical search for singularities was started on the Taylor-Green flow [10], for which the 
results indicate a singularity for a complex value of $t$ but not for real $t$. Experimental results showing vortex reconnection for high-Reynolds number flows inspired a study of singularities for a filament model of two interacting vortex tubes by Siggia [38]. The singularities in this model equation are smoothed out however due to flattening of the vortex cores, as seen in computations of Anderson and Greengard [2], Pumir and Siggia [31] and Shelley, Meiron and Orszag [36]. More recently Kerr [23] presented a new set of computations for this problem which exhibit intensification of vorticity that may indicate singularity formation. Several related studies of singularity formation are in [8,9.16-18,39].

Swirl in axi-symmetric flows amplifies vorticity by stretching, and the axi-symmetry may prevent core flattening, so that such flows seem a likely candidate for singularities. Grauer and Sideris [20]. Meiron and Shelley [26] and Pumir and Siggia [32] have performed computations of axisymmetric flow with swirl that show significant vortex stretching but no singularities within the computational time. In [32] however, an adaptive numerical method, with nonlinear scaling of $x$ and $t$, was used on an asymptotically reduced equation to produce singularities for axi-symmetric flow with swirl. Some critical remarks on the results of [32] are presented in [43].

The present study of axi-symmetric flow with swirl is motivated by the computations of Catflisch, Li and Shelley [12] for axi-symmetric, swirling vortex sheets, with vorticity mainly in the $\hat{z}$ direction. The sheet is destabilized by adding a vortex line on the axis of symmetry with vorticity in the $-\hat{z}$ direction. This basic flow is drawn figuratively in fig. la. Nonlinear computations in [12] then show reconnection of the sheet with itself and with the vortex line.

The basic flow in the present study is a smoothed version of that vortex sheet problem. To avoid geometric singularities at $r=0$ and $r=x$ the flow is put in an annulus $1 \leq r \leq 3$. The vortex line is included through an azimuthal velocity $u_{t}=-I / 2 \pi r$, which can also be thought
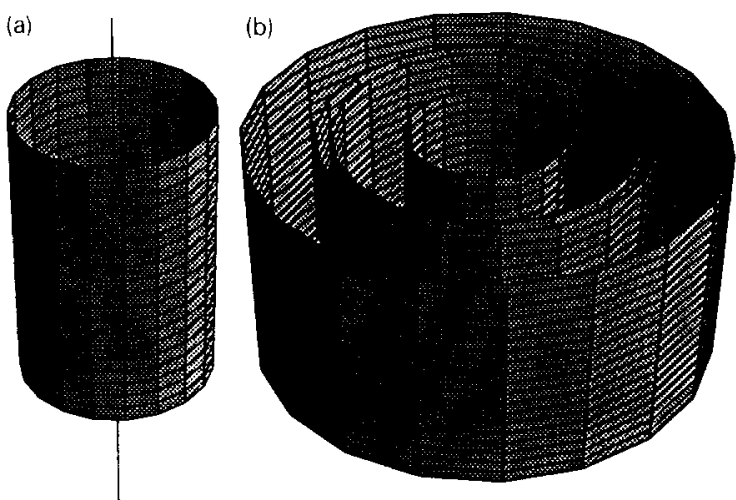

Fig. 1. (a) An axi-symmetric. swirling vortex sheet with a vortex line on the symmetry axis. (b) Smooth swirling fow in an annulus, formed by smoothing out the vortex sheet.

of as vorticity on the boundary $r=1$. The vortex sheet is replaced by a smooth rotational shear layer of small amplitude, so that the resulting basic flow is

$\bar{u}(r)=\left(-\frac{\Gamma}{2 \pi r}+\bar{u}(r)\right) \hat{\theta}$

with vorticity

$\bar{\omega}(r)=r^{\prime} \dot{a}_{1}\left(r \bar{u}_{1}(r)\right) \dot{z}$

which is primarily in the $+\hat{z}$ direction to make the flow unstable. The geometry of this flow is sketched in fig. 1 b and determined in section 4 below.

The Euler solution found here consists of a complex valued velocity field $\boldsymbol{u}$ that is a small perturbation of $\bar{u}$. The velocity field $\boldsymbol{u}$ is periodic in $z$ and contains inward and outward radial jets accompanied by rolls in the $r-z$ plane. The determination of these perturbations is described in section 4, and the real part of the flow is drawn in fig. 2. The singularities found in this study correspond to infinite values of the radial and axial velocity and occur at the centers of these rolls. In contrast the singularities of Pumir and Siggia [32] occur at the tips of the outward jet. 


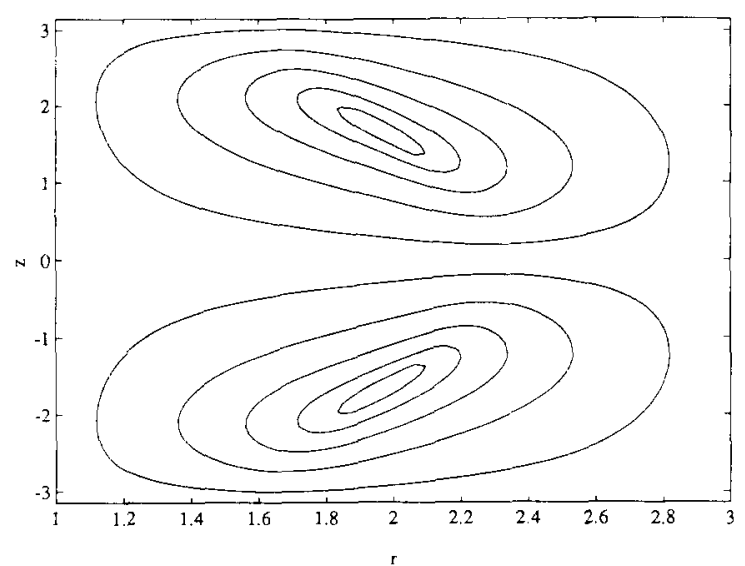

Fig. 2. Streamlines in the $r-z$ plane for the unstable mode. Singularities in complex Euler solutions are found at the centers of these rolls.

The motivation for considering complexvalued velocity fields comes from a generalization of Moore's approximation. Derived by Moore for the Kelvin-Helmholtz problem $[27,28]$, this method has been generalized to additional fluid interface problems by Caflisch, Orellana and Siegel [15], and in section 2 it is formulated as a general approximation for singularity formation. When applied to the Euler equations for axi-symmetric flow with swirl, the velocity $\boldsymbol{u}$ is split into two complex velocities $\boldsymbol{u}_{+}$ and $\boldsymbol{u}_{-}=\overline{\boldsymbol{u}}_{+}$, in which $\boldsymbol{u}_{+}$consists of the nonnegative wavenumber components of $\boldsymbol{u}$. The complex velocity $\boldsymbol{u}_{+}$(as well as $\boldsymbol{u}_{-}$) evolves according to the Euler equations (without any changes), so that Moore's approximation leads to the same Euler equations for axi-symmetric flow with swirl but with complex initial data.

The special solution $u_{+}(r, z, t)$ found here is a traveling wave $u_{+}=u_{+}(r, z-\mathrm{i} \sigma t)$ with an imaginary wave speed, which is motivated by the traveling wave solutions discovered by Siegel $[5,37]$ for Moore's approximation to the Rayleigh-Taylor problem. Singularities move in from the complex $z$-plane at speed $\mathrm{i} \sigma$ and occur physically when they hit the real $z$ line at finite real time $t$. The solution is found through a numerical method that is very accurate but extremely unstable; growth of round off error is controlled by using a multi-precision package MPFUN developed by Bailey $[3,4]$ which allows precision of arbitrarily high order. Round off error levels of $10^{-64}$ and $10^{-128}$ were employed in the computations described below.

The computation is performed for $f_{k}(r)$, the $k$-wavenumber (in $z$ ) component of the radial velocity. There is no singularity in $f_{k}(r)$ for finite $k$; rather singularities are detected through the asymptotic properties of $f_{k}$ as $k \rightarrow \infty$, as in [40]. This procedure is described in section 6 .

The resulting singularity in the radial velocity $u_{r}$ has the form

$u_{r} \cong\left(\eta^{2}-\tau+\mathrm{i} \zeta\right)^{-1 / 3}$,

in which $\eta$ and $\zeta$ are orthogonal spatial coordinates and $\tau$ is a scaled time coordinate, all centered at the singularity. The simplicity of this singularity form may indicate that it is generic for this problem.

The complex-analytic approach to singularities used here follows similar earlier investigations on a variety of simpler problems; including the Kelvin-Helmholtz problem $[14,25,27,28,35]$, the Rayleigh-Taylor problem $[5,29,30,42]$, the Hele-Shaw problem $[22,41]$, and nonlinear hyperbolic or elliptic systems with exactly 2 speeds [11]. For the first two problems, Moore's approximation was shown to give excellent predictions for the time, location and type of singularity formation. For nonlinear hyperbolic (or elliptic) systems, a method was developed for "unfolding singularities" and the generic (stable) types of singularities were found in [11].

\section{Moore's approximation}

As presented here, Moore's approximation is a general method that can be applied to singularity formation problems. It is based on the following consideration of energy cascade and 
inverse cascade due to non-linearities. Since $\mathrm{e}^{i k=}$ $\mathrm{e}^{i l=}=\mathrm{e}^{\mathrm{i}(l+k)=}$, the action of nonlinearity on wavenumbers $k$ and $l$ is to add them to get wavenumber $k+l$. Decompose these interactions into two groups:

(i) energy outflow (forward cascade). If

$\operatorname{sgn}(k)=\operatorname{sgn}(l)$

then $|k+l|>\max (|k|,|l|)$ so that energy is flowing out to higher wavenumbers.

(ii) energy backflow (inverse cascade). If

$\operatorname{sgn}(k)=-\operatorname{sgn}(l)$

then $|k+l|<\max (|k|,|l|)$ so that energy is flowing back to a smaller wavenumber.

A smooth function (analytic in a finite strip) $u(z)$ has very little energy at high wavenumbers. As a singularity forms, however, energy flows out to high wavenumbers. Thus we expect the singularity formation to be dominated by energy outflow which is much stronger than the energy backflow. The approximation here is to neglect energy backflow. This cannot be valid for a steady state, in which outflow and backflow must balance, but it is expected to be at least qualitatively correct for the transient problem of singularity formation.

According to the classification (ii) above, Moore's approximation then amounts to neglecting interactions between positive and negative wavenumbers. This can be formulated mathematically as follows: Suppose that

$$
\begin{aligned}
& u(z)=u_{+}(z)+u_{-}(z), \\
& u_{+}(z)=\sum_{k=1} \hat{u}_{k} \mathrm{e}^{\mathrm{i} k z}, \\
& u(z)=\sum_{k=11} \hat{u}_{k} \mathrm{e}^{i k z}
\end{aligned}
$$

assuming at first that $u$ has no constant term. If $A$ is an operator (linear or non-linear) which is analytic in $u$ and with $A[0]=0$, then Moore's approximation is

$A|u| \cong A|u|+,A|u|$.

The neglected terms all are nonlinearities involving both $u_{+}$and $u$. i.e. both positive and negative wavenumbers.

More generally if

$u-u_{u}+u_{1}+u \quad$.

in which $u_{11}$ is constant in $z$, then Moore's approximation is

$A[u] \cong A\left[u_{4}+u_{n} \mid+A\left[u+u_{n}\right]-A\left[u_{11}\right]\right.$.

This will be applied to the equations for axisymmetric flow with swirl in the next section. Note that the notation $w_{+}$is used sometimes for the sum of all the positive wavenumbers (not including $u_{0}$ ) and other times for the sum of all the non-negative wavenumbers (including $u_{11}$ ).

The validity of this approximation for singularity formation problems has not been rigorously established. For the Kelvin-Helmholtz [35] and Rayleigh-Taylor [5] problems. however, comparisons between solutions of Moore's approximation and of the full problem show excellent agreement for the time, location and type of singularity, as well as the overall shape of the solution. Moreover in the latter problem, the singularities occur at times when the solution has large perturbations in its shape and is fully nonlinear. A related approximation method has been used in a variety of other problems [19.21].

On the other hand Moore's approximation can be easily used to show that singularities do not form in the original problem if they do not form for Moore's approximation, since ignoring the energy backflow biases the problem in favor of singularity formation. For example in [13], the solution of the Birkhoff-Rott equation for the Kelvin-Helmholtz problem was shown to remain smooth as long as the solution of Moore's approximation remains smooth. 


\section{Axi-symmetric flow with swirl}

In this section, the equations for axi-symmetric flow with swirl are formulated, Moore's approximation is applied to them and, finally, traveling wave solutions with imaginary wave speed are investigated.

In primitive variables $\boldsymbol{u}=\left(u_{r}, u_{\theta}, u_{z}\right)$ and $p$ the equations for axi-symmetric flow with swirl are

$\partial_{z} u_{z}+r^{-1} \partial_{r}\left(r u_{r}\right)=0$

$\partial_{t} u_{z}+\boldsymbol{u} \cdot \nabla u_{z}=-\partial_{z} p$

$\partial_{t} u_{r}+\boldsymbol{u} \cdot \nabla u_{r}-r^{-1} u_{\theta}^{2}=-\partial_{r} p$

$\partial_{t} u_{\theta}+\boldsymbol{u} \cdot \nabla u_{\theta}+r^{-1} u_{r} u_{\theta}=0$,

in which $\boldsymbol{u} \cdot \boldsymbol{\nabla}=u_{z} \partial_{z}+u_{r} \partial_{r}$. The vorticity is

$$
\begin{aligned}
\boldsymbol{\omega} & =\left(\omega_{r}, \omega_{\theta}, \omega_{z}\right) \\
& =\left(-\partial_{z} u_{\theta}, \partial_{z} u_{r}-\partial_{r} u_{z}, r^{-1} \partial_{r}\left(r u_{\theta}\right)\right) .
\end{aligned}
$$

\subsection{Moore's approximation}

Denote the system (3.1)-(3.4) as

$E[\boldsymbol{u}]=0$

and set

$\boldsymbol{u}=\boldsymbol{u}_{+}+\boldsymbol{u}_{-}+\boldsymbol{u}_{0}$

in which

$$
\begin{aligned}
& \boldsymbol{u}_{+}=\boldsymbol{u}_{+}(r, z, t)=\sum_{k>0} \hat{\boldsymbol{u}}_{k}(r, t) \mathrm{e}^{\mathrm{i} k z} \\
& \boldsymbol{u}_{-}=\boldsymbol{u}_{-}(r, z, t)=\sum_{k<0} \hat{\boldsymbol{u}}_{k}(r, t) \mathrm{e}^{\mathrm{i} k z} \\
& \boldsymbol{u}_{0}=\overline{\boldsymbol{u}}(r)=\overline{\boldsymbol{u}}_{\theta}(r) \hat{\boldsymbol{\theta}} .
\end{aligned}
$$

Moore's approximation is to replace (3.6) by

$$
E\left[\boldsymbol{u}_{+}+\boldsymbol{u}_{0}\right]+E\left[\boldsymbol{u}_{-}+\boldsymbol{u}_{0}\right]-E\left[\boldsymbol{u}_{0}\right]=0 .
$$

Since $E\left[\boldsymbol{u}_{0}\right], E\left[\boldsymbol{u}_{+}+\boldsymbol{u}_{0}\right]-E\left[\boldsymbol{u}_{0}\right]$ and $E\left[\boldsymbol{u}_{-}+\right.$ $\left.\boldsymbol{u}_{0}\right]-E\left[\boldsymbol{u}_{0}\right]$ consist of all zero, all positive and all negative wavenumbers respectively, then each of them must be zero separately, i.e.

$E\left[\boldsymbol{u}_{+}+\boldsymbol{u}_{0}\right]=0$,

$E\left[\boldsymbol{u}_{-}+\boldsymbol{u}_{0}\right]=0$,

$E\left[\boldsymbol{u}_{0}\right]=0$

The equation (3.14) for $\boldsymbol{u}_{0}$ is satisfied for any $\boldsymbol{u}_{0}$ of the form in (3.10). Moreover if $\boldsymbol{u}$ is real, then $\boldsymbol{u}_{0}$ is real and $\boldsymbol{u}_{-}=\overline{\boldsymbol{u}}_{+}$, so that it suffices to solve (3.12) for $\boldsymbol{u}_{+}$. The corresponding real velocity field is then

$$
\begin{aligned}
\boldsymbol{u} & =\boldsymbol{u}_{0}+\boldsymbol{u}_{+}+\boldsymbol{u}_{-} \\
& =\boldsymbol{u}_{0}+2 \operatorname{Re}\left(\boldsymbol{u}_{+}\right) .
\end{aligned}
$$

\subsection{Traveling wave solutions}

For simplicity redefine the notation so that

$\boldsymbol{u}=\boldsymbol{u}_{0}+\boldsymbol{u}_{+}=\sum_{k=0}^{\infty} \hat{\boldsymbol{u}}_{k}(r, t) \mathrm{e}^{\mathrm{i} k z}$

Following Siegel [37] look for a traveling wave solution with an imaginary wave speed

$$
\begin{aligned}
\boldsymbol{u} & =\boldsymbol{u}(r, z-\mathrm{i} \sigma t) \\
& =\sum_{k=0}^{\infty} \hat{\boldsymbol{u}}_{k}(r) \mathrm{e}^{\mathrm{i} k z+\sigma k t} .
\end{aligned}
$$

Such solutions can also be thought of as consisting of pure growing modes and are partly motivated by a set of solutions for the Hele-Shaw problem derived by Saffman [22, 34]. Such solutions are only possible if $\boldsymbol{u}$ is complex. For such a traveling wave the Euler equations (3.1)-(3.4) become

$$
\begin{aligned}
& \partial_{z} u_{z}+r^{-1} \partial_{r}\left(r u_{r}\right)=0, \\
& -\mathrm{i} \sigma \partial_{z} u_{z}+\boldsymbol{u} \cdot \nabla u_{z}=-\partial_{z} p, \\
& -\mathrm{i} \sigma \partial_{z} u_{r}+\boldsymbol{u} \cdot \nabla u_{r}-r^{-1} u_{\theta}^{2}=-\partial_{r} p
\end{aligned}
$$


$-\mathrm{i} \sigma \dot{d}_{2} u_{H}+\boldsymbol{u} \cdot \nabla u_{\theta}+r^{1} u_{\theta} u_{r}=0$.

Since $u$ will be a perturbation of the basic flow $\bar{u}(r)$ write

$\boldsymbol{u}=\overline{\boldsymbol{u}}+\boldsymbol{u}^{\prime}, \quad p=\bar{p}+p^{\prime}$.

Also to remove the i's denote

$u_{z}^{\prime}=\mathrm{i} U_{z}^{\prime}, \quad K=-\mathrm{i} \dot{d}_{z}$.

The equations for $\boldsymbol{u}^{\prime}$ are then

$r^{\prime} \partial_{r}\left(r u_{r}^{\prime}\right)-K U_{z}^{\prime}=0$,

$\sigma K U_{:}^{\prime}+K p^{\prime}=A$.

$\sigma K u_{r}^{\prime}-2 r^{-1} \bar{u}_{H} u_{H}^{\prime}+\bar{\alpha}_{r} p^{\prime}=B$.

$\sigma K u_{\theta}^{\prime}+\bar{\omega}_{2} u_{r}^{\prime}=C$.

in which the nonlinear terms are

$$
\begin{aligned}
A & =\left(U_{z}^{\prime} K-u_{r}^{\prime} \partial r\right) U_{z}^{\prime}, \\
B & =\left(U_{z}^{\prime} K-u_{r}^{\prime} \partial r\right) u_{r}^{\prime}+r^{-1} u_{t}^{\prime 2}, \\
C & =\left(U_{z}^{\prime} K-u_{r}^{\prime} \partial_{r}\right) u_{\theta}^{\prime}-r^{1} u_{r}^{\prime} u_{\theta}^{\prime} \\
& =U_{z}^{\prime} K u_{\theta}^{\prime}-\omega_{z}^{\prime} u_{r}^{\prime} .
\end{aligned}
$$

The system (3.22) can be simplified to a single second order equation for $u_{r}^{\prime}$ as

$\dot{\partial}_{r}\left[r^{-1} \partial_{r}\left(r u_{r}^{\prime}\right)\right]-\left[K^{2}+\kappa(r)\right] u_{r}^{\prime}=D$.

in which

$\kappa(r)=\frac{2 \bar{u}_{\theta} \bar{\omega}_{z}}{\sigma^{2} r}$

$D=\sigma^{1}\left(\dot{\partial}_{r} A-K B\right)-\frac{2 \bar{u}_{\theta}}{\sigma^{2} r} C$.

The remaining components of velocity and vorticity are expressed in terms of $u_{r}^{\prime}$ as

$u_{z}^{\prime}=\mathrm{i} U_{z}^{\prime}=\mathrm{i} K^{-1}\left(\partial_{r} u_{r}^{\prime}+r^{-1} u_{r}^{\prime}\right)$,

$u_{t \prime}^{\prime}=\sigma{ }^{1} K^{-1}\left(-\bar{\omega}_{z} u_{r}^{\prime}+C\right)$. $\omega_{r}^{\prime}=-\mathbf{i} K u_{H}^{\prime}$.

$\omega_{z}^{\prime}=r^{1}{ }^{\prime}{ }_{r}\left(r u_{\theta}^{\prime}\right)$.

$\omega_{H}^{\prime}=-\mathrm{i} K^{-1}\left(\kappa u_{r}^{\prime}+D\right)$.

Expand $u_{r}^{\prime}$ in a Fourier series as

$u_{r}^{\prime}(r, z)=\sum_{k=0}^{\prime} f_{k}(r) \mathrm{e}^{i k z}$

The equations for $f_{k}$ are then from (3.24)

$\left.L_{k} f_{k} \equiv \dot{a}_{r} \mid r{ }^{\prime} \partial_{r}\left(r f_{k}\right)\right]-\left(k^{2}+\kappa\right) f_{k}=D_{k}$

for $k=1,2, \ldots$ and $1 \leq r \leq 3$, in which $D_{k}$ is the $k$ th Fourier coefficient of $D$. The boundary conditions are

$f_{k}(r=1)=f_{k}(r=3)=0$.

The infinite system of ODE's (3.29), (3.30) are the equations that will be solved numerically to produce a solution of the axi-symmetric Euler equations with swirl.

Several significant advantages have been gained by restricting attention to solutions with only non-negative wavenumbers $k$ and to traveling wave solutions:

(i) The original equations in three space and one time variable, have been reduced to one space and one wavenumber.

(ii) Since $D$ is purely quadratic in $u^{\prime}$, and since the wavenumbers in $u_{r}^{\prime}$ are all positive, then

$D_{k}=\varphi_{k}\left(f_{1}, \ldots, f_{k}\right)$.

This shows that the coupling between the equations in (3.29) is only in one direction. Therefore any finite number of components $f_{1}, \ldots, f_{k}$ can be computed without reference to $f_{i}$ for $l>k$, i.e. without truncation error.

(iii) As a special case of (ii), $D_{1} \equiv 0$, so that $f_{1}$ satisfies

$L_{1} f_{\mathrm{s}}=0$ 
This is exactly the equation for a linearly unstable mode $u_{r}(r, z, t)=f_{1}(r) \mathrm{e}^{\mathrm{i} z+o t}$ around the steady swirling flow $\overline{\boldsymbol{u}}=\bar{u}_{\theta}(r) \hat{\boldsymbol{\theta}}$ with growth rate $\sigma$. A suitable steady flow and growing mode will be determined in the next section.

(iv) The traveling wave speed $\sigma$ is thus determined from the linear eigenvalue problem (3.32) and is independent of the amplitude of the traveling wave. This remarkable property of upper analytic traveling waves was discovered by Michael Siegel [37] and is in marked contrast to usual nonlinear traveling wave problems, for which the determination of the wave speed is the most nonlinear part.

(v) Since $\boldsymbol{u}=\boldsymbol{u}(r, z-\mathrm{i} \sigma t)$ is a traveling wave with imaginary wave speed, singularities travel in the complex $z$ plane at speed $\mathrm{i} \sigma$. Thus a singularity with imaginary component $\rho=-\operatorname{Im}(z)$ at $t=0$ will hit the real $z$ line at $t=\rho / \sigma$. The singularities position should also depend on $r$, i.e. $\rho=\rho(r)$, and the first singularity will occur for $r$ which minimizes $\rho(r)$, as indicated in fig. 3 . Thus a singularity will occur at a real, finite space and time point, if the computed initial data $\boldsymbol{u}(r, z)$ has a singularity at any complex value of $z$.

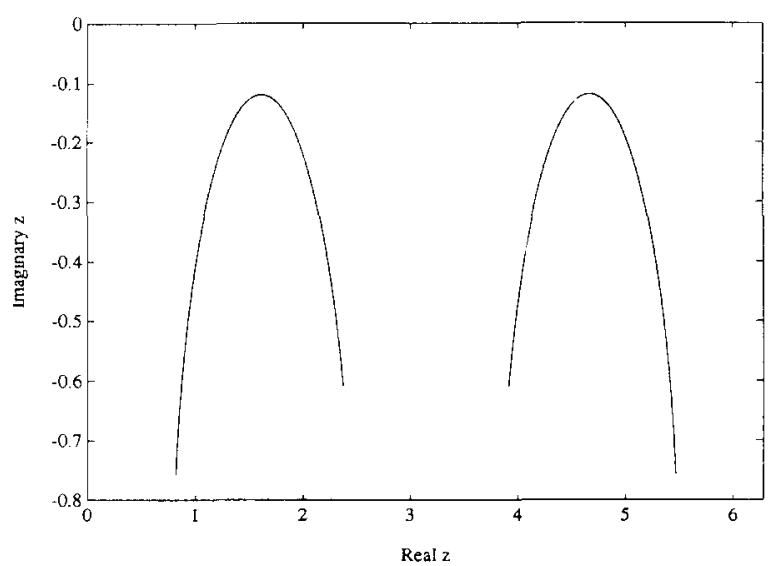

Fig. 3. Singularity positions in the complex $z$ plane. For each value of radius $r$, there is a symmetric pair of singularities on these curves.

\section{The steady flow and its unstable mode}

The eigenvalue problem (3.32) for $f_{1}$ and $\sigma$ may be difficult to solve in general. Here we formulate a special direct procedure by which $f_{1}$, $\sigma$ and the steady state $\bar{u}_{\theta}(r)$ are determined with a minimum of numerical approximation. Rewrite (3.32) and (3.25) as

$\kappa=\frac{(L-1) f_{1}}{f_{1}}$

and

$\kappa=\frac{2}{\sigma^{2} r} \bar{u}_{\theta} \bar{\omega}_{z}=\frac{1}{\sigma^{2} r^{3}} \partial_{r}\left(r \bar{u}_{\theta}\right)^{2}$,

in which $L=\partial_{r}\left(r^{-1} \partial_{r} r\right)$. Then

$\bar{u}_{\theta}(r)=r^{-1}\left(\sigma^{2} \int_{r_{1}}^{r} r^{3} \kappa(r) \mathrm{d} r+\left[r_{1} \bar{u}_{\theta}\left(r_{1}\right)\right]^{2}\right)^{1 / 2}$,

$\bar{\omega}_{z}(r)=\frac{\sigma^{2} r \kappa}{2 \bar{u}_{\theta}}$.

Therefore if $f_{1}(r)$ and $\sigma$ are specified, then (4.1) determines $\kappa$ in terms of $f_{1}$ and (4.3) determines $\bar{u}_{\theta}$ and $\bar{\omega}_{z}$. The only restriction is that the quantity inside the square root for $\bar{u}_{\theta}$ must be positive. To insure this a special choice of $f_{1}$ and $\sigma$ is made: The unstable mode $f_{1}$ is a smoothed version of the unstable mode $\tilde{f}_{1}$ for a vortex sheet problem, and $\sigma$ is the corresponding growth rate.

Consider the problem of a cylindrical swirling vortex sheet at $r=r_{0}$ and with a background rotating flow inside an annulus $r_{1}<r<r_{2}$. The circulation is $\Gamma_{1}$ inside the sheet and $\Gamma_{2}$ outside the sheet, i.e.

$\tilde{\boldsymbol{u}}_{\theta}= \begin{cases}\Gamma_{1} / 2 \pi r, & r_{1}<r<r_{0}, \\ \Gamma_{2} / 2 \pi r, & r_{0}<r<r_{2} .\end{cases}$

According to Rayleigh's criterion, if $\left|\Gamma_{1}\right|>\left|\Gamma_{2}\right|$ this swirling flow is unstable, and the $k=1$ unstable mode has radial velocity

$\tilde{u}_{r}(r, z, t)=a \tilde{f}(r) \mathrm{e}^{\mathrm{i} z+\sigma t}$. 
The amplitude $a$ is arbitrary, and the velocity profile $\tilde{f}(r)$, derived in appendix $\mathrm{B}$, is given by

$\tilde{f}(r)= \begin{cases}b_{1} I_{1}(r)-b_{2} K_{1}(r), & r_{1}<r<r_{01}, \\ b_{3} I_{1}(r)-b_{4} K_{1}(r), & r_{01}<r<r_{2},\end{cases}$

in which $I_{1}$ and $K_{1}$ are the modified Bessel functions [1],

$b_{1}=\sigma K_{11}\left(K_{11} I_{10}-I_{11} K_{10}\right){ }^{\prime}$,

$b_{3}=\sigma K_{12}\left(K_{12} I_{10}-I_{12} K_{10}\right)^{\prime}$.

$b_{2}=b_{1} l_{11} / K_{11}$,

$b_{4}=b_{3} l_{12} / K_{12}$,

and the notation

$I_{1 i}=I_{1}\left(r_{i}\right), \quad K_{1 i}=I_{1}\left(r_{i}\right)$

is used. The growth rate for this mode is given by

$\sigma= \pm \sqrt{(b / c) G / r_{0}^{2}}$

in which

$b=-\left(K_{11} I_{10}-I_{11} K_{10}\right)\left(K_{12} I_{10}-I_{12} K_{10}\right)$,

$c=K_{11} I_{12}-I_{11} K_{12}$,

$G=\left(\Gamma_{1}^{2}-\Gamma_{2}^{2}\right) /(2 \pi)^{2}$.

For the unstable mode in the smooth problem, take $f_{1}$ to be a smoothed version of $\tilde{f}$, i.e.

$f_{1}(r)=\alpha(r) I_{1}(r)-\beta(r) K_{1}(r)$

in which $\alpha$ goes smoothly from $b_{1}$ to $b_{3}$ and $\beta$ goes smoothly from $b_{2}$ to $b_{4}$. Set

$\alpha(r)=\frac{1}{2}\left(b_{1}+b_{3}\right)+\frac{1}{2}\left(b_{3}-b_{1}\right) \phi(r)$,

$\beta(r)=\frac{1}{2}\left(b_{2}+b_{4}\right)+\frac{1}{2}\left(b_{4}-b_{2}\right) \phi(r)$,

in which $\phi$ goes smoothly from -1 to 1 as $r$ goes from $r$, to $r$, i.e. $\phi\left(r_{1}\right)=-1, \quad \phi\left(r_{2}\right)=1$.

If $\phi$ is monotone, then $f_{1}>0$ for $r_{1}<r<r_{2}$ since $\tilde{f}>0$.

In order that the ratio $(L-1) f_{1} / f_{1}$ makes sense, several conditions must be met. Using

$\dot{\partial}_{r} I_{1}=I_{0}-r^{-1} I_{1}$.

$\partial_{r} K_{1}=-K_{0}-r{ }^{1} K_{1}$,

calculate

$$
\begin{aligned}
(L-1) f_{1}= & \left(\phi_{r r}-r{ }^{\mathrm{l}} \phi_{,}\right) \\
& \times \frac{1}{2}\left[\left(b_{3}-b_{1}\right) I_{1}-\left(b_{4}-b_{2}\right) K_{1}\right] \\
& +\phi_{r}\left[\left(b_{3}-b_{1}\right) I_{11}+\left(b_{4}-b_{2}\right) K_{11}\right] .
\end{aligned}
$$

Since $f_{1}$ vanishes at $r=r_{1}$ and $r=r_{2}$, also $L_{1} f_{1}$ must be zero there: it suffices to require

$\phi_{r r}=\phi_{r}=0 \quad$ at $r=r_{1}$ and $r=r_{2}$.

A function $\phi$ satisfying (4.13) and (4.16) can be chosen. in terms of an odd function $\psi_{11}$. as

$\phi(r)=c_{0} \psi_{0}(\rho)-\left(c_{1} \rho+c_{2} \rho^{3}\right)$

in which $\rho \in(-1,1)$ for $r \in\left(r_{1}, r_{2}\right)$ and

$c_{0}=\left(\psi_{0}(1)-\psi_{0}^{\prime}(1)+\frac{1}{3} \psi_{0}^{\prime \prime}(1)\right)^{\prime}$.

$c_{1}=c_{0}\left(\psi_{0}^{\prime}(1)-\psi_{0}^{\prime \prime}(1)\right)$.

$c_{2}=c_{10} \psi_{11}^{\prime \prime}(1) / 6$.

A suitable choice for $\rho(r)$ and $\psi_{0}(\rho)$ is

$\rho(r)=\frac{r-r_{01}}{r_{2}-r_{1}}\left(\frac{r-r_{2}}{r_{1}-r_{0}}+\frac{r-r_{1}}{r_{2}-r_{11}}\right)$.

$\psi_{0}(\rho)=\tanh (\rho / \delta)$

so that 
$\psi_{0}^{\prime}=\left(1-\psi_{0}^{2}\right) / \delta$,

$\psi_{0}^{\prime \prime}=-2 \psi_{0}^{\prime} \psi_{0} / \delta$

$\psi_{0}^{\prime \prime}=-2 \psi_{0}^{\prime}\left(1-3 \psi_{0}^{2}\right) / \delta^{2}$

$\psi_{0}^{\prime \prime \prime}=-2\left(-8 \psi_{0}+12 \psi_{0}^{3}\right) \psi_{0}^{\prime} / \delta^{3}$,

with the arbitrary parameter $\delta$ representing the thickness of the rotational shear layer (smoothed vortex sheet) at $r=r_{0}$.

In particular the limiting values of $\kappa=(L-1) f_{1} / f_{1}$ at $r=r_{1}, r_{2}$ are computed from l'Hôpital's rule as follows: Using $\left(K_{1} I_{0}+\right.$ $\left.K_{0} I_{1}\right)(r)=r^{-1}$

$\partial_{r} f_{1}\left(r=r_{i}\right)= \begin{cases}b_{1}\left(K_{11} r_{1}\right)^{-1}, & i=1, \\ b_{3}\left(K_{12} r_{2}\right)^{-1}, & i=2,\end{cases}$

$\partial_{r}(L-1) f_{1}$

$$
=\frac{1}{2} \phi_{r r r}\left(K_{12} I_{11}-I_{12} K_{11}\right) \begin{cases}K_{12}^{-1} b_{3}, & i=1, \\ K_{11}^{-1} b_{1}, & i=2,\end{cases}
$$

so that

$$
\begin{aligned}
& \kappa\left(r_{1}\right)=\frac{1}{2} r_{1} \phi_{r r r}\left(K_{12} I_{11}-I_{12} K_{11}\right) K_{11} b_{3} /\left(K_{12} b_{1}\right) \\
& \kappa\left(r_{2}\right)=\frac{1}{2} r_{2} \phi_{r r r}\left(K_{12} I_{11}-I_{12} K_{11}\right) K_{12} b_{1} /\left(K_{11} b_{3}\right),
\end{aligned}
$$

in which

$\phi_{r r r}=\psi^{\prime \prime \prime} \rho^{\prime 3}$

at $r=r_{1}$ or $r=r_{2}$.

To summarize, for any choice of the parameters $r_{1}, r_{0}, r_{2}, \Gamma_{1}, \Gamma_{2}, \delta$ and $a$, satisfying $r_{1}<r_{0}<$ $r_{2}$ and $\left|\Gamma_{1}\right|>\left|\Gamma_{2}\right|$, there is an unstable mode $f_{1}$ and growth rate $\sigma$ given by (4.11) and (4.9). The corresponding steady flow $\bar{u}_{\theta}(r)$ can be computed through a single integration from (4.3), in which the function $\kappa(r)=(L-1) f_{1} / f_{1}$ is given by $(4.15)$ for $r_{1}<r<r_{2}$ and by (4.22) at the endpoints $r=r_{1}$ or $r=r_{2}$. There is a restriction that $\bar{u}_{\theta}$ is real, i.e the quantity inside the square root in (4.3) must be non-negative, which is the case for the choice of parameters used below. In the following computations, the modified Bessel functions are evaluated through a series expansion, and the integral in (4.3) is evaluated using 4th-order accurate finite difference-like formulas.

\section{Numerical method}

The numerical method for solving the system of 2-point boundary value problems (3.29), (3.30) is summarized as follows:

\subsection{Discretization}

A 4th-order finite difference method is used for the $r$-dependence, with centred differences in the interior and one-sided differences at the boundaries. The extra terms near the edges are first removed by Gaussian elimination, so that the resulting linear system can be solved with a pentadiagonal solver. The number $N_{r}$ of points in $r$ is 512,1024 or 2048 in the computations presented here.

The computation is for a finite number $N_{z}$ of wavenumbers, with $N_{z}=64$ in the computations. The nonlinear terms $\hat{D}_{k}$ on the right side of (3.29) are evaluated by an accelerated method involving both direct and pseudospectral sums, as described in appendix C. As pointed out in section 3 , there is no truncation error in the restriction to finite $N_{z}$. Also since $D$ is quadratic, anti-aliasing (i.e. padding with enough zeroes) completely eliminates aliasing error from the pseudo-spectral part of the computation. The resulting complexity of the algorithm is $N_{r} N_{z}^{3 / 2} \log N_{z}$.

\subsection{Round-off error}

The $k$-dependence of the system (3.29) turns out to be extremely unstable so that roundoff error is amplified and may ruin the computation. This is similar to the amplification of roundoff error found in vortex sheet computations by 
Krasny, where the computation was stabilized by filtering [25] or smoothing [24]. Filtering is ineffective here because the problem is not periodic in $r$ (this could perhaps be overcome using a Chebyshev representation); smoothing is too crude to allow detection of singularities.

The roundoff error problem was overcome through high precision computation using the multiple precision package MPFUN developed by David Bailey at NASA Ames Research Center $[3,4]$. This package allows arbitrary specified precision and includes a translator that converts a standard Fortran program into one that calls special subroutines for multiplication and other built-in functions. For $N_{r}=512$, quadruple precision with roundoff-error $\varepsilon_{r} \cong 10^{-28}$, available on an IBM 3090 running AIX was found to be sufficient. For $N_{r}=1024$ and 2048, roundoff error $\varepsilon_{r}=10^{64}$ and $10^{-128}$ respectively was used.

The instability with respect to $k$, as well as its elimination for sufficiently small $\varepsilon_{r}$, is illustrated in fig. 4. This graph shows $\max _{r} f_{k}(r)$ vs. $k$ computed with $N_{r}=512$ for single precision $\left(\varepsilon_{r} \cong 10^{7}\right)$, double precision $\left(\varepsilon_{r} \cong 10^{14}\right)$, quadruple precision $\left(\varepsilon_{r} \cong 10^{-2 \kappa}\right)$ and multiple precision using MPFUN with $10^{-64}$, the last two of which are indistinguishable.

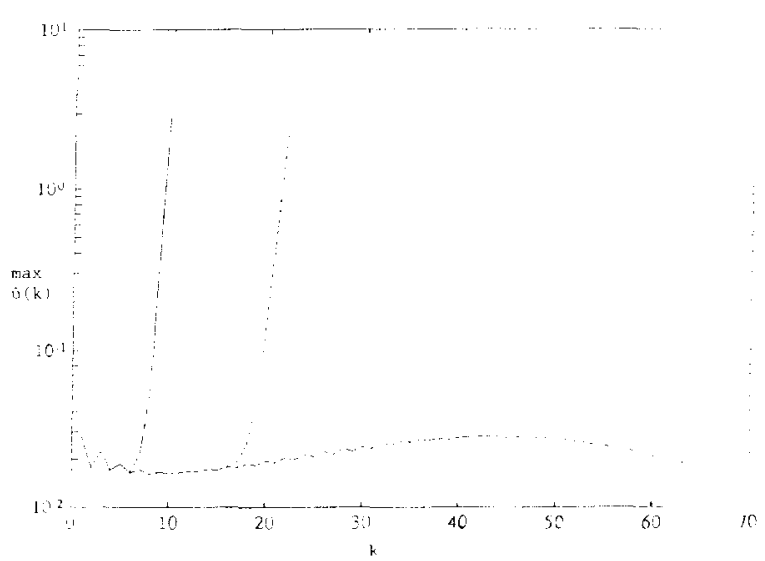

Fig. 4. Effect of roundoff error on the solution for $N_{k}=512$. Graph is $\max _{r} \hat{u}^{\prime \prime \prime}(k)$ for single precision $(-)$; double precision $(-\cdot)$; quadruple precision $(--)$; precision $10^{\text {tit }}(\cdot \cdot)$. The last two are indistinguishable, showing sufficient accuracy for this value of $N_{k}$.

\subsection{Choice of parameters}

The computational results here are for the following choice of parameters in the basic flow $\bar{u}_{i)}\left(0\right.$ th mode) and lst mode $f_{1}$, as described in section 4 :

$r_{1}=-1.0 . \quad I_{2}=0.1$.

$\delta=0.3, \quad a=0.028$,

$r_{1}=1, \quad r_{11}=2, \quad r_{2}=3$

This put the singularity time close to (1 to give a good range for the Fourier coefficients.

\section{Computational results and singularities}

The numerical computation described above for the traveling wave determines the solution at a fixed time $t=0$.0. From these results, a singularity time $t^{*}$ is found through the singularity detection method described in section 6.2 below. Moreover, the solution can be reconstructed at any time $t_{10}$ from the numerical data at time $t=0$ through the traveling wave formula in (3.17): i.e. through multiplication of the $k$ th Fourier coefficient by a factor $e^{i k h e}$

\subsection{Spatial velocity profiles}

Fig. 5 shows the velocity profile at a time $t=t_{*}-1.0$ before the singularity. The radial and axial velocities $u$, and $u_{\text {. }}$ (poloidal components) are illustrated through the level surfaces of the real part of stream function $\%$, satisfying

$\operatorname{Re}\left(u_{,}, u_{z}\right)=\frac{1}{r} \operatorname{Re}\left(-\psi_{z}, \psi_{r}\right)$

in fig. 5a. This shows an outward radial jet accompanied by $r-z$ rolls that are tilted toward the center of the jet. In figs. $5 b$ and $5 c$, contour plots of the corresponding real functions $\Omega=r u_{,}$ and $\zeta=-r \omega_{0}$ are drawn.

The profile of the solution at (approximately) the singularity time $t=t_{*}$ is presented in fig. 6 in 

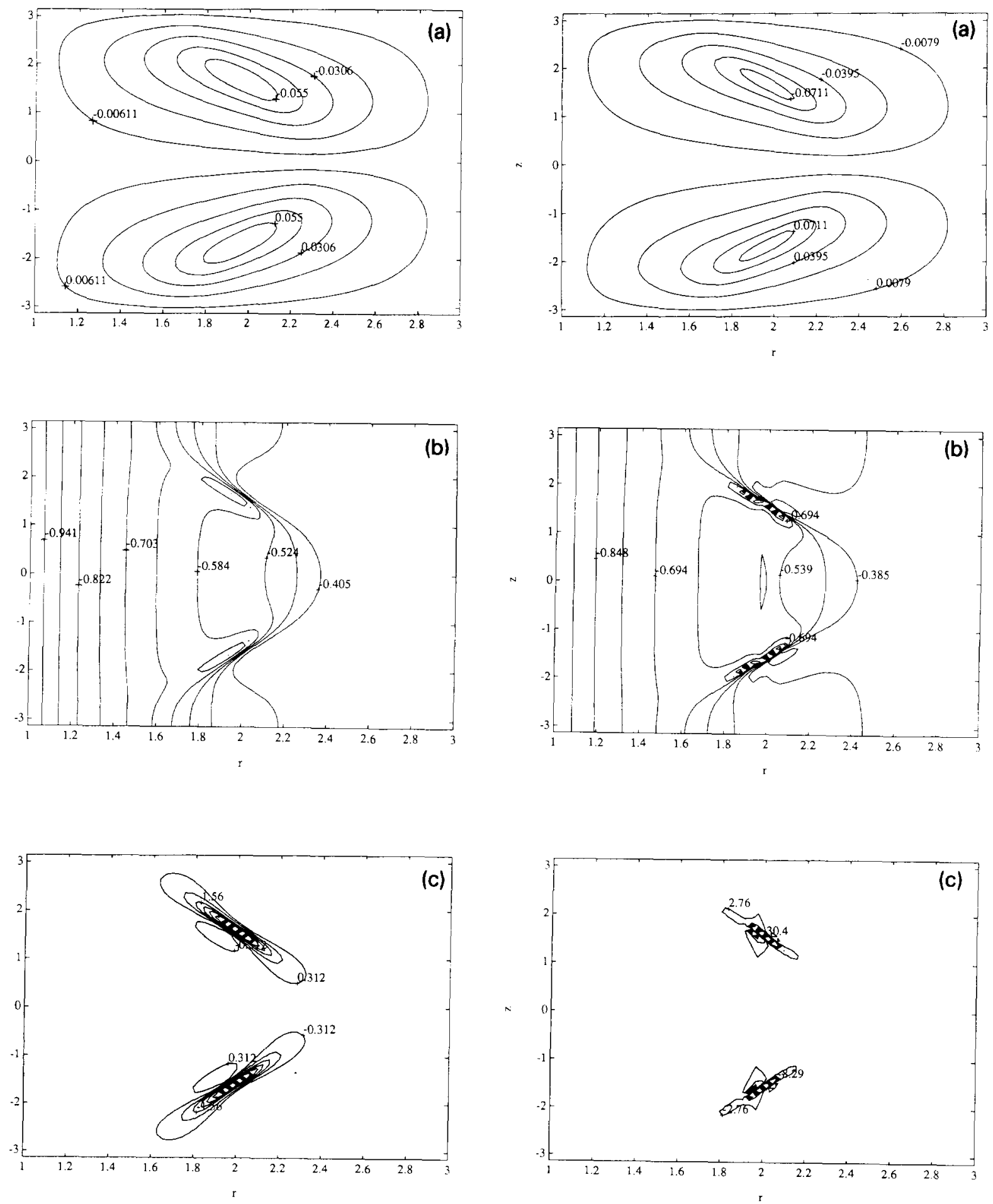

Fig. 5. Contour plots of (a) $\psi$, (b) $\Omega$, (c) $\zeta$ at $t=t^{*}-1.0$, in which $t^{*}$ is the numerically determined singularity time.

Fig. 6. Same as fig. 5, but at the singularity time $t=t^{*}$. 
the same way. This shows that the singularities occur at the centers of the rolls, which will also be verified through analysis of the Fourier coefficients. This is in contrast to the computations of Pumir and Siggia [32] and of Grauer and Sideris [20], in which the greatest amplification of vorticity and possible singularities occur at the tip of the outward jet.

The functions $\psi$ and $\zeta$ are odd while $\Omega$ is even. The maximum values of $|\zeta|$ and $|\Omega|$ are plotted versus time in fig. 10. These values are approximate, since they are formed through a sum of the 64 Fourier modes, as determined in this computation, each multiplied by $\mathrm{e}^{i k t}$ to get the correct $t$ dependence. As a result, the values cannot grow faster than $\mathrm{e}^{6, \mathrm{~s} t}$ and are necessarily finite at the singularity time $t_{* *}$. Nevertheless, over a time interval of length 2.0, the value of the azimuthal vorticity $\omega_{t}$ is seen to increase by a factor of more than 20 , while the circulation $\Omega$ increases by a factor of less than 3. This suggests that $\omega_{i}$ blows up, while $\Omega$ remains bounded. Clean evidence for the blowup of $\omega_{t}$ follows from the singularity analysis of the next two subsections. A similar asymptotic fit to the Fourier coefficients of $\Omega$, which could provide clean evidence that it does not blow up, was not successful.

\subsection{Numerical detection of singularities}

Following the results for the spatial profiles of velocity and the general formulation of section 3 , two singularities are expected to occur at positions

$z_{+}(r)=-\mathrm{i} \rho_{1} \pm \rho_{2}$.

Near each of these singularities the structure of the radial velocity $u_{r}$ is sought in the form

$u_{r}(z, r) \simeq c_{1} \mathrm{e}^{i u_{z}}\left(z-z_{ \pm}\right)^{-1-\left(\alpha_{1}-1\left(\alpha_{2}\right)\right.} \equiv$

for $z$ near $z_{ \pm}$. As shown in [40], this implies that the Fourier coefficients $f_{k}$ will have the asymp- totic form

$f_{k} \cong c_{1} k{ }^{\prime \prime} \mathrm{e}^{p_{1} k} \sin \left(c_{2}+\alpha_{2} \log k+\rho_{2} k\right)$

for $k \geqslant 1$. In $(6.2)-(6.4)$ the parameters $\left(c_{1}, c_{2}\right.$ $\left.\alpha_{1}, \alpha_{2}, \rho_{1}, \rho_{2}\right)$ depend on $r$, i.e.

$$
\begin{aligned}
& \left(c_{1}, c_{2}, \alpha_{1}, \alpha_{2}, \rho_{1}, \rho_{2}\right) \\
& =\left(c_{1}, c_{2}, \alpha_{1}, \alpha_{2}, \rho_{1}, \rho_{2}\right)(r) .
\end{aligned}
$$

Following $[5,29,30],\left(c_{1}, c_{2}, \alpha_{1}, \alpha_{2}, \rho_{1}, \rho_{2}\right)$ are determined through a sliding fit: i.e. for each $k$. the parameters are chosen to exactly fit the 6 values $f_{k}, f_{k+1} \ldots f_{k}$. The asymptotic fit is successful if the values $\left(c_{1}, c_{2}, \alpha_{1}, \alpha_{2}, \rho_{1}, \rho_{2}\right)$ are (nearly) independent of the starting index $k$, as well as independent of the discretization size $\mathrm{d} r=2 \pi / N_{r}$.

Fig. 7 shows these 6 parameters as functions of $k$ at $r=2$ for two values $N_{r}=1024$ and 2048 . This shows convergence as $N, \rightarrow$ as well as a successful fit with parameter values that are independent of $k$. Note that $10 \alpha_{2}$ is plotted so that the variations in $\alpha_{2}$ on the graph are actually quite small. The fit is equally good for other values of $r$.

The $r$-dependence of the parameters $\left(c_{1}, \alpha_{1}\right.$. $\left.\rho_{1}\right)$ and $\left(c_{2}, \alpha_{2}, \rho_{2}\right)$ is plotted in figs. $8 \mathrm{a}$ and $8 \mathrm{~b}$. The parameters $\alpha_{1}$ and $\alpha_{2}$ are given by $\alpha_{1}=$ $-\frac{2}{3} \pm 0.01$ and $\alpha_{2}=0.0 \pm 0.01$ independent of $r$. The amplitude term $c_{1}$ is also nearly constant: while the phase $c_{2}$ and the real position $\rho_{2}$ are approximately linear in $r$.

As pointed out in section 3 , the first singularity in time corresponds to the minimum value of the complex position $\rho_{1}(r)$ for $r=r_{\text {min }}$. This is seen to occur at approximately $r=2$. The derivative $\mathrm{d} \rho_{1} / \mathrm{d} r$ is plotted in fig. 9 which shows that $\rho_{1}$ is quadratic in $r$ at $r_{\text {man }}$.

\subsection{Local analysis of the singularities}

Now the time dependence is included by replacing $z$ with $z-\mathrm{i} \sigma t$. Using the above results, 

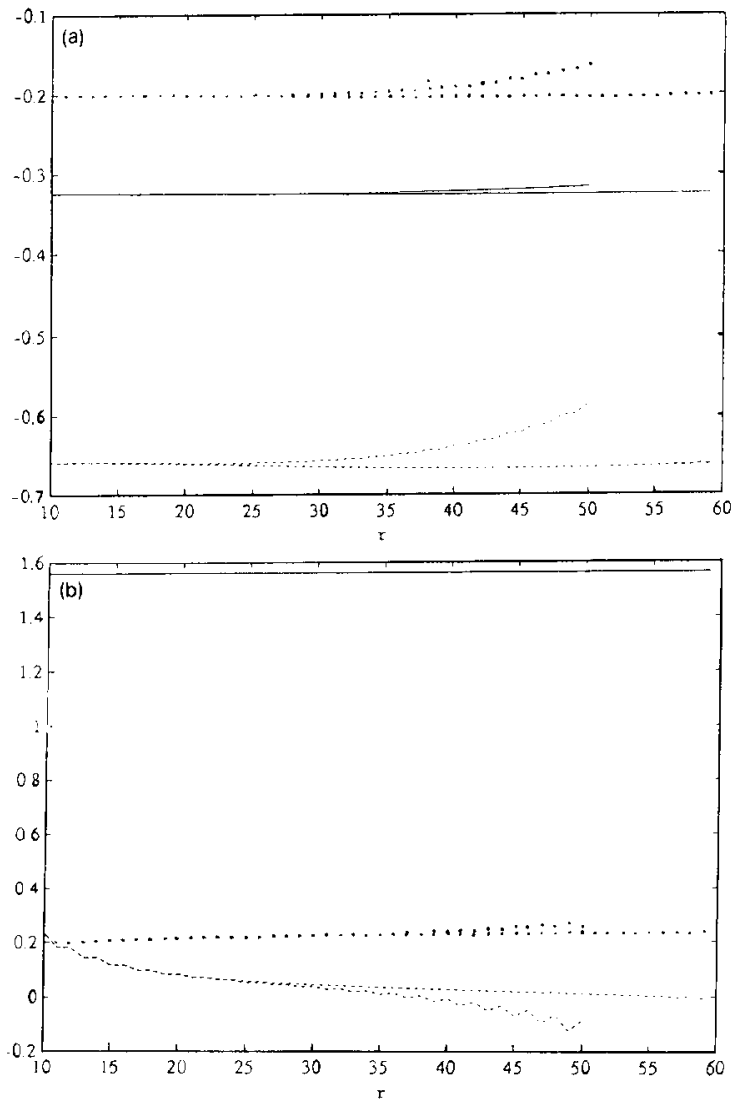

Fig. 7. Results from sliding fit for Fourier coefficients at $r=2.0$ as a function of $k$. In (a) the graphs are of $5 \rho_{1}(-), \alpha_{1}$ $(--)$, and $5 c_{1}(\cdot \cdot)$. In (b) the graphs are of $\rho_{2}(-), 10 \alpha_{2}(--)$, and $c_{2}(\cdot)$. For each parameter the shorter curve is for $N_{r}=1024$, while the longer is for $N_{r}=2048$. The fit is judged to be successful since the results are nearly independent of $k$ and $N_{r}$.

for $r$ near $r_{\min }$ and $z$ near $z_{+}+\mathrm{i} \sigma t$ we approximate

$c_{1}=\bar{c}_{1}, c_{2}=\bar{c}_{2}$,

$\rho_{1}=\bar{\rho}_{1}+\bar{\rho}_{11}\left(r-r_{\min }\right)^{2}$,

$\rho_{2}=\bar{\rho}_{2}+\bar{\rho}_{21}\left(r-r_{\min }\right)$,

$\alpha_{1}-\mathrm{i} \alpha_{2}=-\frac{2}{3}$.

Define new orthogonal variables centered at the real singularity position and time by
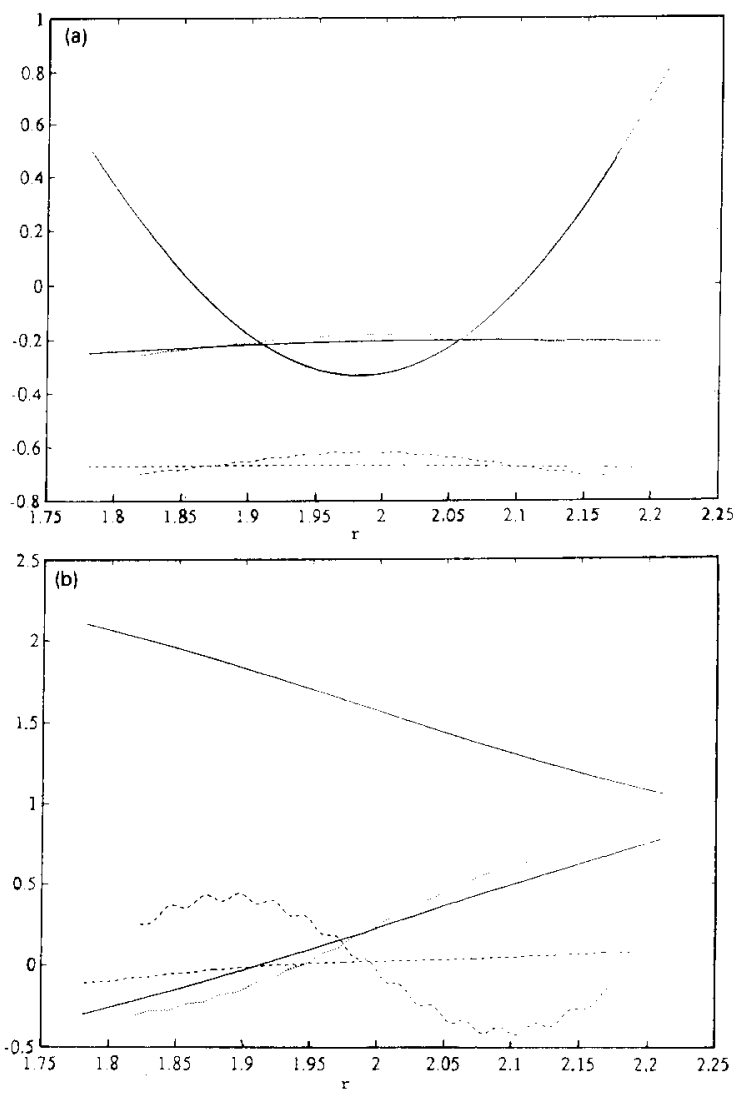

Fig. 8. Singularity parameters as a function of $r$ from the sliding fit. In (a) the graphs are of $5 \rho_{1}(-), \alpha_{1}(--)$, and $5 c_{1}$ $(\cdot)$. In (b) the graphs are of $\rho_{2}(-), 10 \alpha_{2}(--)$, and $c_{2}(\cdot)$. For each parameter the shorter curve is for $N_{r}=1024$, while the longer is for $N_{r}=2048$. Note that $\alpha_{2} \approx 0.0 \pm 0.1$.

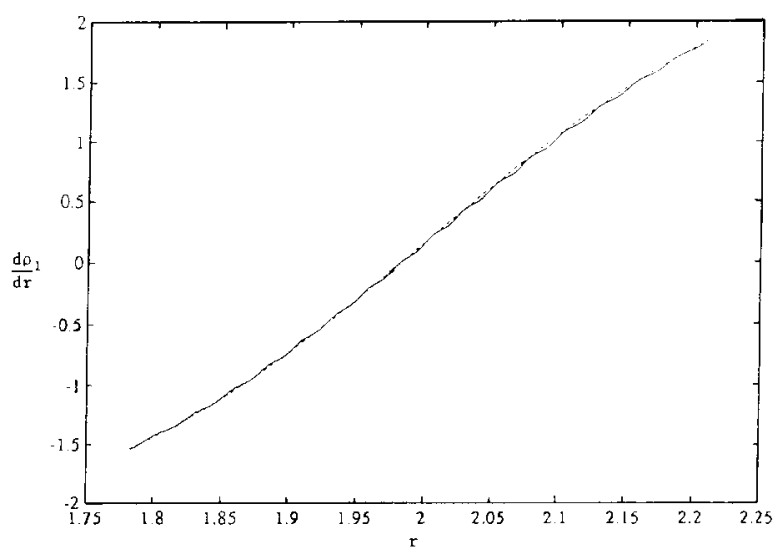

Fig. 9. $\mathrm{d} \rho_{1} / \mathrm{d} r$ as a function of $r$, showing that $\rho_{1}$ is a nondegenerate quadratic at its minimum. 
$\tau=\sigma t-\bar{\rho}_{1}$.

$\zeta=\left(z-\bar{\rho}_{2}\right)-\bar{\rho}_{21}\left(r-r_{\min }\right)$,

$\eta=\sqrt{\bar{\rho}_{11}}\left[\left(r-r_{\min }\right)+\bar{\rho}_{21}\left(z-\bar{\rho}_{2}\right)\right]$.

The spatial variable $\eta$ runs in the direction of the major semi-axis of the (nearly) elliptic rolls in the spatial profile of figs. 5 and 6 ; while $\zeta$ runs in the direction of the minor semi-axis. The form of the singularity in $(6.3)$ is then

$u_{r}(z, r, t) \cong c \xi^{1 / 3}$.

in which the singularity variable $\zeta$ is

$\xi=\eta^{2}-\tau-\mathrm{i} \zeta$

This remarkably simple form for the singularity may indicate that the results are generic for the Euler equations.

The corresponding stream function is

$\psi(z, r, t) \cong c \xi^{2 / 3}$.

This shows that singularity occurs at the centers of oval-shaped rolls which are flattening as the singularity forms.

\section{Conclusions}

The computations and analysis presented above demonstrate development of singularities from smooth initial data for complex-valued Euler solutions. A clean analysis of the singularity is possible because of the unusual nature of the computation: The degrees of freedom have been reduced by looking for a traveling wave, and computational errors are minimized since there are no truncation or aliasing errors in the computation of a finite number of Fourier components. Most important, the computation is for the Fourier coefficients, none of which blow up at the singularity. Instead, the singularity is found by analyzing the asymptotics of the Fourier coefficients.

Although the motivation for considering such solutions is through Moore's approximation, the complex-valued velocity fields constructed here are solutions of the usual Euler equations. On the other hand. there is no evidence that the singularities in these complex solutions have any relevance for real solutions; i.c., the singularities found under Moore's approximation could be spurious.

The significance of these results is that they provide simple examples of solutions with singularities, as well as a reasonable conjecture for the location and structure of singularities in real flows. We also conjecture that errors in Moore's approximation are small, so that the combination $\boldsymbol{u}=\boldsymbol{u}_{0}+2 \operatorname{Re} \boldsymbol{u}_{+}$(which is real) will be an approximate solution of the Euler equations.

Although this conjecture has not been verified, several consistency conditions are met by the complex solutions: First, although the real energy $\int|\boldsymbol{u}|^{2} \mathrm{~d} \boldsymbol{x}$ is not conserved for a complex solution, it is finite at the singularity computed here. Second for a traveling wave solution, time $t$ and axial position $z$ are linearly related. The time integral of vorticity is thus related to a spatial integral of vorticity, which is a velocity. Therefore the Beale-Kato-Majda result [7] that $\int^{\prime} \max |\omega| \mathrm{d} t \rightarrow \infty$ at a singularity should imply $|\boldsymbol{u}| \rightarrow \infty$ at this singularity. in agreement with the results above. Third the circulation $\Omega$ remains bounded, as indicated in fig. 10 .

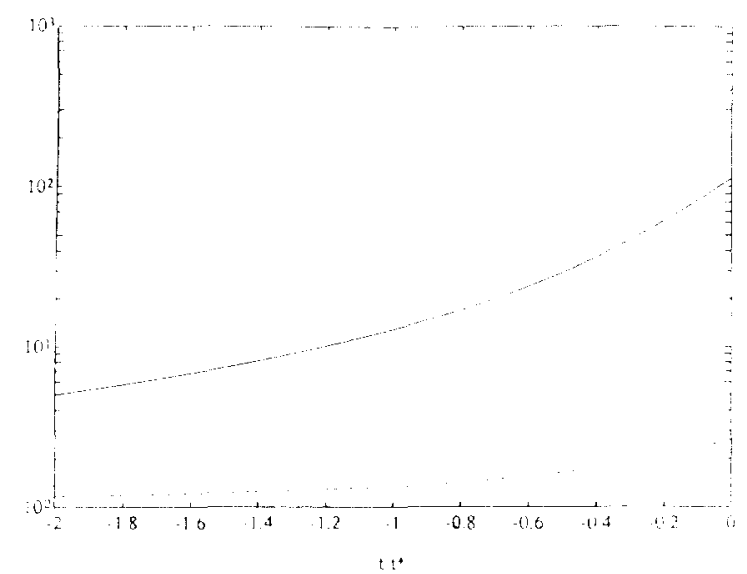

Fig. 10. Maximum values of $\zeta(-)$ and $\Omega(--)$ as a function of t. computed using the Fourier sum of 64 modes 
Appendix A. Alternative formulations of the Euler equations

The Euler equations (3.1)-(3.4) for axisymmetric flow with swirl can be written in two other useful representations.

\section{A.1. Stream function and vorticity}

Define circulation $\Omega=r u_{\theta}$, azimuthal vorticity $\zeta=-r \omega_{\theta}$ and stream function $\psi$ with

$\left(u_{r}, u_{z}\right)=r^{-1}\left(-\partial_{z} \psi, \partial_{r} \psi\right)$.

Denote

$\boldsymbol{u} \cdot \boldsymbol{\nabla}=u_{r} \partial_{r}+u_{z} \partial_{z}$,

$D^{2}=r \partial_{r}\left(r^{-1} \partial_{r}\right)+\partial_{z}^{2}$.

Then (3.1)-(3.4) is equivalent to

$\left(\partial_{\boldsymbol{t}}+\boldsymbol{u} \cdot \boldsymbol{\nabla}\right) \boldsymbol{\Omega}=0$,

$\left(\partial_{t}+u \cdot \nabla\right)\left(r^{-2} \zeta\right)=-r^{-4} \partial_{z}\left(\Omega^{2}\right)$,

$D^{2} \psi=\zeta$.

If Moore's approximation is applied to this system and a traveling wave solution is sought, the equation for the Fourier coefficients $\Psi_{k}^{(r)}=$ $-\mathrm{i} \hat{\psi}(r, k)$ is

$r \frac{\mathrm{d}}{\mathrm{d} r} r^{-1} \frac{\mathrm{d}}{\mathrm{d} r} \Psi_{k}-\left(k^{2}+\kappa\right) \Psi_{k}=E_{k}$,

in which $\kappa$ is defined by (3.25) and $E_{k}$ is a nonlinear function of $\Psi_{0}, \ldots, \Psi_{k-1}$ and their derivatives.

\section{A.2. Clebsch variables}

For a restricted class of axi-symmetric initial data, the flow can be described through a special form of Clebsch variables, as pointed out to us by $M$. Shelley and M. Vishik. Define a bracket $[\cdot, \cdot]$ by $[\alpha, \beta]=-\frac{1}{r}\left(\alpha_{r} \beta_{z}-\alpha_{z} \beta_{r}\right)$

which satisfies the Jacobi identity

$[[\alpha, \beta], \gamma]+[[\beta, \gamma], \alpha]+[[\gamma, \alpha], \beta]=0$.

Denote $\rho=\Omega^{2}, \zeta=-r \omega_{\theta}$ and let $D^{2}$ and $\psi$ be defined as above. Suppose that $\zeta$ can be expressed as

$\zeta=-r^{2}[\alpha, \rho]$

for some function $\alpha(r, z)$. If this is true initially it will remain true, and the Euler equations (3.1)(3.4) for axi-symmetric flow with swirl are equivalent to the following system:

$\rho_{t}=[\psi, \rho]$,

$\alpha_{t}=[\psi, \alpha]+\frac{1}{2 r^{2}}$,

$D^{2} \psi=-r^{2}[\alpha, \zeta]$.

If the initial data for such a flow is smooth, then $\alpha$ will stay bounded for all time.

The $1 / 2 r^{2}$ term in the equation for $\alpha$ introduces an artificial time dependence which may be removed by setting $\alpha=\beta+t \gamma$. The equations for $\rho, \beta, \gamma$ and $\psi$ are then

$\rho_{i}=[\psi, \rho]$,

$\gamma_{t}=[\psi, \gamma]$,

$\beta_{t}=[\psi, \beta]-\gamma+\frac{1}{2 r^{2}}$,

$D^{2} \psi=-r^{2}[\beta, \rho]$,

with the condition that

$[\gamma, \rho]=0$.

Since

$\frac{\mathrm{d}}{\mathrm{d} t}[\gamma, \rho]=[\psi,[\gamma, \rho]]$ 
the condition (A.10) is maintained by the evolution

A traveling wave $(\rho, \beta, \gamma, \psi)(r, z-\mathrm{i} \sigma t)$ solves the equations

$-\mathrm{i} \sigma \rho_{z}=|\psi, \rho|$,

$-\mathrm{i} \sigma \gamma_{z}=\lfloor\psi, \gamma \mid$.

$-\mathrm{i} \sigma \beta_{z}=[\psi, \beta]-\gamma+\frac{1}{2 r^{2}}$,

$D^{2} \psi=-r^{2}[\beta, \rho]$.

Use of the Jacobi identity (A.5) shows that

$[\gamma, \rho]=-\frac{i}{\sigma r} \psi_{r}[\gamma, \rho]$

so that $[\gamma, \rho]=0$ as required.

The steady swirling flow $\bar{u}$ in section 3 corresponds to

$\rho=\bar{\rho}(r)=\left\lfloor\left. r \bar{u}_{\theta}(r)\right|^{2}\right.$,

$\gamma=\bar{\gamma}(r)=\left(2 r^{2}\right)^{\prime}$.

$\beta=\psi=0$.

The equation for a traveling wave $\psi=\mathrm{i} \psi(r, z-$ $\mathrm{i} \sigma t)$ as in section 3 is then, after some manipulation.

$r{ }_{r} r{ }^{\prime} \partial, \psi-\left(K^{2}+\kappa\right) \Psi=A$

in which $K=-i x_{x}$ and the $A$ is quadratically nonlinear in $\Psi$. In particular the $k=1$ Fourier component $\hat{\Psi}_{1}$ satisfies

$r \dot{\partial}{ }_{r} r^{-1} \partial_{r} \psi-(1+\kappa) \psi=0$

which is identical to (3.32) for $f_{1}=r^{-1} \hat{\Psi}_{1}$. Since both the solution $\boldsymbol{u}$ of section 3 and the Clebsch solution $\psi$ are determined by their 0 th and first Fourier modes and since these can be chosen to coincide, then every upper analytic traveling wave solution $\boldsymbol{u}$ as in section 3 has a Clebsch representation.

On the other hand the class of Clebsch solutions is not structurally stable. i.e. if $\boldsymbol{u}$ is a
Clebsch solution with $\nabla \rho=0$ at some point, then small perturbations of $\boldsymbol{u}$ need not be Clebsch. First if $\nabla \rho=0$ but $\zeta \neq 0$ (which will generally be truc after a small perturbation), then clearly there can be no such solution $\alpha$ of (A.7). The other solvability condition for (A.7) is an integral condition around closed loops of the vector field $\left(-\rho_{x}, \rho_{r}\right)$ in $(r, z)$. Inside such a loop there must be a singular point of the field at which $\nabla \rho=0$.

The lack of structural stability implies that if a real flow $\tilde{u}$ can be constructed from the present complex flow as $\tilde{\boldsymbol{u}} \approx \boldsymbol{u}_{0}+2 \operatorname{Re}\left(\boldsymbol{u}_{+}\right)$, then it need not be Clebsch. This is significant since for a Clebsch flow with $\psi \cong c \xi^{23}$, as in (6.10), either $\rho$ or $\alpha$ must blowup. Since these are both bounded for an initially smooth Clebsch solution, a real flow $\tilde{\boldsymbol{u}}$ can be close to $\boldsymbol{u}_{0}+2 \operatorname{Re}\left(\boldsymbol{u}_{+}\right)$only if $\tilde{\boldsymbol{u}}$ is not Clebsch.

\section{Appendix B. Linear instability of an axi- symmetric vortex sheet with swirl in an annulus}

Consider the steady flow generated by an axisymmetric vortex sheet $r=r_{11}$ with a background rotation in an annulus $r_{1}<r<r_{2}$. The circulation is taken to be $\Gamma_{1}$ inside the sheet and $\Gamma_{2}$ outside the sheet. The potential for the unperturbed flow is then

$\Phi_{1}=\theta \Gamma_{1} / 2 \pi \quad r_{1}<r<r_{n}$.

$\Phi_{2}=\theta \Gamma_{2} / 2 \pi, \quad r_{11}<r<r_{2}$.

For the perturbed flow there is a free surface at $r=r_{11}+\xi(z, t)$ and the potential is

$\Phi_{1}=\theta \Gamma_{1} / 2 \pi+\phi_{1}(r, z, t), \quad r_{1}<r<r_{11}+\varepsilon$.

$\Phi_{2}=\theta I_{2} / 2 \pi+\phi_{2}(r, z, t) . \quad r_{11}+\xi<r<r_{2}$.

Following [12,33], the linearized equations for $\phi_{1}, \phi_{2}$ and $\xi$ are $\nabla^{2} \phi_{1}=\nabla^{2} \phi_{2}=0$ (in cylindrical coordinates) with linearized free boundary conditions 
$\phi_{1 r}=\phi_{2 r}=\xi_{t}$,

$\phi_{1 t}-r_{0}^{-3}\left(\Gamma_{1} / 2 \pi\right)^{2} \xi=\phi_{2 t}-r_{0}^{-3}\left(\Gamma_{2} / 2 \pi\right)^{2} \xi$

on $r=r_{0}$ and

$\phi_{1 r}\left(r=r_{1}\right)=\phi_{2 r}\left(r=r_{2}\right)=0$.

The $k$ th mode for these equations is

$\phi_{1}=\left[b_{1} I_{0}(k r)+b_{2} K_{0}(k r)\right] \mathrm{e}^{\mathrm{i} k z+\sigma t}$,

$\phi_{2}=\left[b_{3} I_{0}(k r)+b_{4} K_{0}(k r)\right] \mathrm{e}^{\mathrm{i} k z+\sigma t}$,

$\xi=\mathrm{e}^{\mathrm{i} k z+\sigma t}$,

in which the coefficients are

$b_{1}=\sigma k^{-1} K_{11}\left(K_{11} I_{10}-I_{11} K_{10}\right)^{-1}$,

$b_{3}=\sigma k^{-1} K_{12}\left(K_{12} I_{10}-I_{12} K_{10}\right)^{-1}$,

$b_{2}=b_{1} I_{11} / K_{11} \quad b_{4}=b_{3} I_{12} / K_{12}$,

with $I_{10}=I_{1}\left(k r_{0}\right)$, etc. The growth rate is

$\sigma= \pm \sqrt{(b / c) G / r_{0}^{2}}$

in which

$c=k^{-2}\left(K_{11} I_{12}-I_{11} K_{12}\right)$,

$b=-\left(K_{11} I_{10}-I_{11} K_{10}\right)\left(K_{12} I_{10}-I_{12} K_{10}\right)$,

$G=\left(\Gamma_{1}^{2}-\Gamma_{2}^{2}\right) /(2 \pi)^{2}$.

Since $K$ is decreasing and $I$ is increasing, then $c>0$, and $b>0$. Therefore the steady flow is unstable exactly if $G>0$, which is precisely Rayleigh's criterion for this flow.

\section{Appendix C. An accelerated method}

The nonlinear terms $D_{k}$ in (3.29) are the Fourier components of the function $D$ defined in (3.26). Since $D$ is quadratic and $k$ is non-negative, $D_{k}$ is a finite convolution
$D_{k}=\sum_{l=1}^{k-1} a_{l}^{(k)} a_{k-l}^{(k)}$,

in which $a_{l}^{(k)}$ is linear in $f_{l}$ for each $k$. A direct computation of the sums (C.1) for each $k \leq N_{z}$ would require $N_{z}^{2}$ operations. Since this is not an initial value problem a standard pseudo-spectral formulation does not work. Here we formulate an accelerated method using $N_{z}^{3 / 2}$ operations.

Choose an increasing sequence of number $M_{1}$, $M_{2}, \ldots, M_{n}$, with $M_{n}=N_{z}$. Suppose that the computation of the modes $k \leq M_{i}$ have been completed. For $M_{i}<k \leq M_{i+1}$ split the sum $D_{k}$ in (C.1) into two parts $D_{k}=D_{k}^{(1)}+D_{k}^{(2)}$ in which $D_{k}^{(1)}$ includes all terms with both $l \leq M_{i}$ and $k-l \leq M_{i}$ and $D_{k}^{(2)}$ contains the remaining terms. For each $i$ the terms in $D_{k}^{(1)}$ involve only $k^{\prime} \leq M_{i}$ so are already known. Thus $D_{k}^{(1)}$ for $M_{i}<k \leq M_{i+1}$ can all be computed together by a pseudo-spectral method, requiring only $M_{i+1} \log M_{i+1}$ operations. The remaining terms $D_{k}^{(2)}$ must be determined sequentially in $k$ and are computed by direct sums, requiring $\left(M_{i+1}-\right.$ $\left.M_{i}\right)^{2}$ steps. The total operation count is

$N_{\mathrm{Op}}=\sum_{i=1}^{n} M_{i} \log M_{i}+\left(M_{i+1}-M_{i}\right)^{2}$.

An optimal choice is $M_{i}=i^{2}, n=N_{z}^{1 / 2}$ for which $N_{\text {Op }}=\mathscr{O}\left(N_{z}^{3 / 2} \log N_{z}\right)$.

\section{References}

[1] M. Abramowitz and I.A. Stegun, Handbook of Mathematical Functions (Dover, 1970).

[2] C.R. Anderson and C. Greengard, The vortex ring merger problem at infinite Reynolds number, Commun. Pure Appl. Math. 42 (1989) 1123-1139.

[3] D.H. Bailey, Automatic translation of fortran programs to multi-precision, RNR Technical Report RNR-90-025 (1991).

[4] D.H. Bailey, Mpfun: A portable high performance multiprecision package. RNR Technical Report RNR-90022 (1991).

[5] G.R. Baker, R.E. Caflisch and M. Siegel, Singularity formation during Rayleigh-Taylor instability J. Fluid Mech. (1993), to appear. 
[6] C. Bardos and S. Benachour, Domaine d'analycite des solutions de l"equation d'Euler dans un ouvert de $\mathbb{R}^{\text {". }}$ Annali della Scuola Normale Superiore di Pisa IV, 4 (1977) 647-687.

[7] J.T. Beale, T. Kato, and A. Majda. Remarks on the breakdown of smooth solutions for the 3D Euler equations, Comm. Math. Phys. 94 (1984) 61-66.

[8] J.B. Bell and D.L. Marcus. Vorticity intensification and transition to turbulence in the three-dimensional Euler equations, Commun. Math. Phys. 147 (1992) 371-394.

$[9 \mid$ A. Bhattacharjee and X. Wang, Finite-time vortex singularity in a model of three-dimensional Euler flows, Phys. Rev. Lett. 69 (1992) 2196-2199.

[10] M.E. Brachet, D. Meiron, S. Orszag, B. Nickel, R. Morf and U. Frisch, Small-scale structure of the TaylorGreen vortex, J. Fluid Mech. 130 (1983) 411-452.

[11] R.E. Caflisch. N. Ercolani and T.Y. Hou, Multi-valued solutions and branch point singularities for nonlincar hyperbolic systems, Commun. Pure Appl. Math. 46 (1993) 453-499

[12] R.E. Caflisch, Xiaofan Li and M.J. Shelley, The collapse of an axi-symmetric swirling vortex sheet. Nonlinearity (1993), to appear.

[13] R.E. Caflisch and O.F. Orellana, Long time existence for a slightly perturbed vortex sheet, Commun. Pure Appl. Math. 39 (1989) 807-838.

[14] R.E. Caflisch, O.F. Orellana, Singularity formulation and ill-posedness for vortex sheets, SIAM J. Math. Anal. 20 (1989) 293-307.

[15] R.E. Caflisch, O.F. Orellana and M. Siegel, A localized approximation for vortical flows, SIAM J. Appl. Math. 50 (1990) 1517-1532.

[16] S. Childress. G.R. lerley, E.A. Spiegel and W.R. Young. Blow-up of unsteady two dimensional Euler and Navier-Stokes solutions having stagnation point form. J. Fluid Mech. 203 (1989) 1-22.

[17] A. Chorin, Estimates of intermittency, spectra and blowup in developing turbulence, Commun. Pure Appl. Math. 24 (1981) 853-856.

[18] A. Chorin, The evolution of a turbulent vortex, Commun. Math. Phys. 83 (1982) 517-535.

[19] P. Constatin and L.P. Kadanoff, Dynamics of a complex interface. Physica D 47 (1991) 450-460).

[20] R. Grauer and T. Sideris, Numerical computation of 3D incompressible ideal fluids with swirl, Phys. Rev. Lett. 25 (1991) 3511-3514.

121] J.M. Greene and I.C. Percival, Hamiltonian maps in the complex plane, Physica D 3 (1981) 530-548.

(22) S.D. Howison, J.R. Ockendon and A.A. Lacey, Singularity development in moving-boundary problems. Quart. J. Appl. Math. 38 (1985) 343-360.

[23] R.M. Kerr, Evidence for a singularity of the threedimensional, incompressible Euler equations, 1992.

[24] R. Krasny, Desingularization of periodic vortex sheet roll-up. J. Comput. Phys. 65 (1986) 292-313.
[25] R. Krasny. A study of singularity formation in a vortex sheet by point-vortex approximation. J. Fluid Mech. 167 ( 1986 ) $65-93$.

[26] D.I. Meiron and M.J. Shelley, personal communication (1992).

127| D.W. Moore, The spontaneous appearance of a singu larity in the shape of an cvolving vortex sheet. Proc. $R$ Soc. London A 365 (1979) 105-119.

[28] D.W. Moore, Numerical and analytical aspects of Helmholtz instability, in: Theoretical and Applied Mechanics. Niordson and Olhoff, eds. Proc. XVI ICTAM (NorthHolland, 1985) pp. 629-633.

|29| D.A. Pugh, Development of vortex sheets in Boussinesy flows - Formation of singularities. Ph.D. thesis, Imperiat College, 1989.

[30] D.A. Pugh and S.J. Cowley, On the formation of an interface singularity in the rising 2D Boussinesq bubble, J. Fluid Mech. (1993), to appear.

[31] A. Pumir and E.D. Siggia. Collapsing solutions to the 3D Euler equations, Phys. Fluids A 2 (1990) 220.

1.32| A. Pumir and F.D. Siggia, Development of singular solutions to the axisymmetric Euler equations. Phys. Fluids A 4 (1992) 1472-1491

[33] R. Rotunno, A note on the stability of a cylindrical vorlex sheet. J. Fluid Mech. 87 (1978) 761-771

[34] P.G. Saffman, Exact solutions for the growth of fingers from a flat interface between two fluids in a porous medium or Hele-Shaw cell. Quart. J. Appl. Math. 12 (1959) 146-150.

[35] M.J. Shelley, A study of singularity formation in vortex sheet motion by a spectrally accurate vortex method, J. Fluid Mech. 244 (1992) 493-526.

[36] M.J. Shelley, D.I. Meiron and S.A. Orszag, Dynamical aspects of vortex reconnection of perturbed anti-parallel vortex tubes. J. Fluid Mech. 246 (1993) 61.3-6.52.

1.37| M. Siegel. An analytical and numerical study of singularity formation in the Rayleigh-Taylor problem. Ph.D. thesis, NYU, 1989.

[38] E.D. Siggia, Collapse and amplification of a wortex filament. Phys. Fluids 28 (1985) 794-8015.

[39] I.T. Stuart. Nonlinear Euler partial differential equations: Singularities in their solution, Symposium in honor of C.C. Lin, 1989.

[40] C. Sulem. P.L. Sulem and H. Frisch. Tracing complex singularities with spectral methods. J. Comput. Phys. 50 (1983) $138-161$.

[41] S. Tanveer, Evolution of Hele-Shaw interface for small surface tension, Philos. Trans. R. Soc. L.ondon (1992), to appear.

[42] S. Tanveer. Singularities in the classical RayleighTaylor flow: formation and subsequent motion, Proc. R. Soc. (1992), to appear.

[43] X. Wang and A. Bhattacharjee, Is there a finite-time singularity in axisymmetric Euler Hows? 1991 